\title{
ENSEÑANZA DE LAS FUNCIONES EXPONENCIALES EN LA ESCUELA SECUNDARIA. ASPECTOS DIDÁCTICOS Y COGNITIVOS
}

\author{
Autor: Diana Patricia Sureda Figueroa. Mail: patri.sureda@gmail.com \\ Tipo:Tesis doctoral \\ Director: Dra. María Rita Otero. Mail: rotero@exa.unicen.edu.ar \\ Universidad: Universidad Nacional del Centro de la Provincia de Buenos Aires. Tandil. \\ Buenos Aires. Argentina \\ Nombre de la carrera: Doctorado en Enseñanza de las Ciencias. Mención Matemática \\ Fecha de defensa: 20 de junio de 2012
}

La tesis aborda el problema de la enseñanza de la función exponencial con sentido (Bosch et al., 2006) en la escuela secundaria, y de su conceptualización. Así, el trabajo se apoya en dos referenciales: uno didáctico, la teoría antropológica de lo didáctico (TAD) propuesta por Chevallard (1999, 2001, 2004, 2007, 2009), y otro cognitivo, la teoría de los campos conceptuales (TCC) de Vergnaud (1990, 1996, 2005, 2007, 2010). Pues, mientras el marco teórico didáctico brinda sustento a las decisiones relativas a la actividad de estudio e investigación (AEI) en los procesos de topogénesis, cronogénesis y mesogénesis, el marco cognitivo permite el análisis de la conceptualización en el aprendizaje. Un punto importante es que en ambas teorías el concepto de situación tiene el carácter de tarea.

Dado que el dispositivo AEI permite estudiar una organización matemática con sentido a partir del estudio de una "situación del mundo" a la que se tiene que dar respuesta, se diseñó una AEI compuesta por doce situaciones problemáticas, tres conjuntos de tareas y una evaluación. Después de una prueba piloto, la AEI fue readaptada e implementada en dos cursos de cuarto año (15-16 años). Se analizaron los protocolos, se tomaron decisiones sobre el ajuste de la AEI propuesta, que debido al nuevo diseño curricular debió considerar también la reubicación de los contenidos en quinto año, y se implementó en dos cursos de quinto año (16-17 años). En total se obtuvo la resolución de 121 alumnos clase a clase, lo que hace un total de 1.440 resoluciones. La recolección sistemática de los protocolos resultó indispensable para acceder a las primeras estrategias formuladas por los estudiantes, las cuales son imprescindibles para el estudio de la conceptualización. Cada intervención se registró mediante un audio general. La implementación demandó dos meses y medio de clases en una escuela de la ciudad que atiende a sectores urbanos medios. Allí se llevaron a cabo el estudio piloto y las cuatro implementaciones.
Para hacer posible la implementación de una AEI en la escuela secundaria, era necesario instalar lo que Chevallard denomina la pedagogía de la investigación en la clase de Matemáticas. En esta pedagogía requiere modificar radicalmente los papeles del profesor, del alumno y de sus lugares en la clase. Así, el lugar del alumno, antes reducido a la aplicación de técnicas previamente enseñadas por el profesor, requería modificarse en una dirección que exige tomar decisiones, asumir la responsabilidad del propio aprendizaje, pensar con otros, etc. Esta modificación en la topogénesis del grupo de clase, por ser una construcción didáctica y cognitiva-afectiva que comprendía nuevas responsabilidades para cada integrante del grupo de clase, requirió un esfuerzo sostenido en el tiempo. Un dispositivo funcional al desarrollo de una clase de Matemática con estas características se materializó en un Acta de Compromiso y Estudio en Matemática (Otero, 2007), mediante la cual se formularon las nuevas normas de estudio, una vez que fueron consensuadas por el grupo de clase. Estas pautas permitieron tanto llevar a cabo la AEI, a través del trabajo autónomo de los alumnos, como cumplir con las restricciones institucionales y sociales de acreditación.

La nueva forma de gestionar el estudio molestó al principio a los alumnos, pues ellos no lograban lidiar con la incertidumbre y la ansiedad que les ocasionaba el tener que tomar decisiones para resolver los problemas sin conocer las respuestas. Pero, hacia el final, la mayor parte de ellos habían aceptado el desafío, y a medida que trascurría la AEI fueron mejorando su desempeño. Hubo otros estudiantes que desde el principio mostraron predisposición para resolver las situaciones y aseguraron que era la primera vez que les iba bien en Matemática. Al terminar la AEI, la mayor parte de los alumnos terminaron remarcando la importancia de permitirles pensar por sí mismos y equivocarse. 
Por otra parte, el análisis de las resoluciones de los alumnos, que se realizó a partir de la TCC, mostró la estrecha relación entre la conceptualización, los sistemas de representación (SR) y los invariantes operatorios (IO) de los estudiantes. También permitió reconocer un proceso de conceptualización de la función exponencial, que comienza en las respuestas totalmente lineales y se va modificando progresivamente en una dirección primero no lineal, y finalmente exponencial. Esta descripción muestra que entre la primera y la última etapa, las respuestas de los estudiantes en la misma situación, y dependiendo del sistema de representación, están guiadas por invariantes operatorios diferentes. Es decir, esquemas diferentes, a veces lineales, a veces exponenciales. Esto muestra que, cuando el conocimiento de un campo conceptual (CC) es incipiente, coexisten esquemas contradictorios entre sí para el mismo concepto. Pero, cuando el estudiante solo estaba en posesión de esquemas lineales, los utilizaba coherentemente en todos los sistemas de representación.
Esto ha sido magistralmente tratado en la TCC por Gérard Vergnaud, quien formuló la definición de concepto como un triplete en el que los SR tienen un papel central, aunque no excluyente. Esto resuelve el problema de la reducción de la matemática a un lenguaje, lo cual es falso, y habitualmente escuchado, a la vez que permite contemplar el papel innegable de los SR en Matemática y la complejidad que supone el dominio de un CC en esta área, pues los conceptos aparecen en distintos marcos (geométrico, analítico, funcional, etc.), los cuales tienen cada uno asociados sus propios SR. En consecuencia, no existe "el esquema exponencial", sino una variedad de esquemas exponenciales que son diferentes según el SR que se esté utilizando. Así, el dominio pleno del CC de las funciones exponenciales deberá involucrar en su enseñanza los diferentes sistemas de representación ligados al concepto.

\title{
INFLUENCIA DE LAS NN. TT. EN EL DOCENTE UNIVERSITARIO DE TOPOGRAFÍA. ESTUDIO DE CASOS
}

\author{
Autor: Manuel José León Bonillo \\ Tipo:Tesis doctoral \\ Directores: Roque Jiménez Pérez y Bartolomé Vázquez Bernal \\ Departamento: Didáctica de las Ciencias y Filosofía \\ Universidad: Universidad de Huelva \\ Programa: Investigación en la Enseñanza y el Aprendizaje de las Ciencias Experimentales, \\ Sociales y Matemáticas \\ Fecha de presentación: 30 de enero de 2012
}

Esta tesis doctoral es consecuencia de un trabajo previo de investigación de acción de aula realizado entre alumnos universitarios, resultando, como parte de las posibles perspectivas futuras, la indagación de la influencia de las nuevas tecnologías en el profesorado de la asignatura de Topografía (León y Aguaded, 2009).

A esta ampliación o ramificación del estudio primigenio, hay que añadirle la adaptación de la asignatura al EEES y su posterior implantación como asignatura de grado para el curso 2011/12.

La investigación se ha llevado a cabo a partir del estudio de casos, concretamente de dos profesores. Destacamos que se ha realizado para el nivel universitario, en el área de conocimiento de la ingeniería, donde los profesores se encuentran por su formación técnica alejados del conocimiento teórico de la Enseñanza de las Ciencias.

El estudio de casos se ha realizado a partir del análisis cualitativo de distintos datos, recogidos a su vez con diferentes herramientas, destacando el estudio cualitativo de grabaciones de aula a partir del visionado directo de los vídeos, sin transcripción previa mediante AQUAD (Huber y Gürtler, 2004).

El objetivo principal es estudiar las influencias del empleo de las NN. TT. en el desarrollo profesional de estos profesores, así como detectar, aislar y reflexionar 
sobre los obstáculos que impedían o impiden al profesorado progresar en su desarrollo profesional.

Esta investigación, además, posee un segundo interés didáctico divulgativo en esta área no familiarizada con la investigación en la Enseñanza de las Ciencias, al tiempo de generar el hábito de la investigación didáctica en escuelas técnicas superiores y promover el desarrollo profesional.

$\mathrm{Al}$ tratar de resumir los principales bloques de esta investigación, debemos mencionar que los núcleos centrales de nuestro marco teórico consisten y están interrelacionados con:

- El constructivismo social, como entorno y paradigma colaborativo de evolución en la investigación profesional.

- La hipótesis de progresión del conocimiento profesional, como eje vertebrador y camino que seguir en las indagaciones del estudio.

- El conocimiento profesional, como base fundamental de todo profesor para poder desarrollar sus labores docentes, tanto desde el punto de vista teórico como práctico.

- El desarrollo profesional, como exigencia ética y social de la buena práctica profesional y resultado de un proceso dinámico evolutivo del conocimiento profesional.

- Los obstáculos en el desarrollo profesional, como impedimentos del conocimiento profesional que obstaculizan el desarrollo profesional, los cuales hay que identificar, analizar y reflexionar.

El marco metodológico seguido y de acuerdo con el marco teórico puede resumirse en un trabajo de investigación que estudia el desarrollo profesional del profesor universitario, a partir de un estudio de casos, metodología cualitativa desde un enfoque del desarrollo curricular.

Es decir, realizamos un estudio cualitativo interpretativo a partir de grabaciones de vídeos y registros etnográficos, entre otros instrumentos. Siguiendo una hipótesis de progresión del conocimiento profesional en función del desarrollo profesional y curricular que se experimenta a causa de la introducción de nuevas herramientas pedagógicas. Los datos recopilados serán reducidos a un sistema de categorías para el análisis, y concretados en el marco curricular, subdividido en tres dimensiones de estudio: dimensión técnica, práctica y crítica.

El análisis de la práctica ha sido estudiado a partir de instrumentos de primer orden tales como los registros etnográficos de la práctica de aula, el visionado y la codificación directa en diferentes etapas con la ayuda de un programa de análisis cualitativo y la evolución de artefactos como los apuntes, ejercicios de clase y programas de la asignatura.

El análisis de datos quedaría resumido en los siguientes apartados:

- Concepciones iniciales y finales declaradas.

- Análisis de la reflexión.

- Análisis de la práctica.

Los resultados se han subdivididos en:

- Interacción entre la reflexión y la práctica.

- Obstáculos e influencias de las NN. TT.

En el apartado conclusiones e incidiendo en el doble interés de la investigación y el aspecto didáctico divulgador, hemos visto oportuno incluir unas líneas dedicadas a las perspectivas de la investigación y a las implicaciones didácticas de esta.

En resumen (León, 2012), aunque puede ser considerado como una réplica de otros tantos estudios realizados con fines similares, destacamos la originalidad de:

- Realizarse en el nivel universitario.

- Un estudio de caso con amplio desarrollo temporal (Vázquez-Bernal et al., 2012), presente y futuro.

- En docentes sin una formación pedagógica formal.

- El análisis de vídeos sin necesidad de una transcripción previa. 


\title{
LOS INSTRUMENTOS DE FÍSICA EN LOS MANUALES Y EN LOS GABINETES DEL SIGLO XIX EN ESPAÑA. ESTUDIO DE CASO: EL GABINETE DEL IES «P. SUÁREZ»DE GRANADA
}

\author{
Autor: Jesús Sánchez Tallón \\ Tipo:Tesis doctoral \\ Director: Manuel Fernández González \\ Departamento: Didáctica de las Ciencias Experimentales \\ Universidad: Universidad de Granada \\ Programa: Didáctica de las Ciencias Experimentales \\ Fecha de presentación: 19 de diciembre de 201 I
}

Esta tesis trata sobre la enseñanza de la física en el siglo XIX en España, prestando especial atención al desarrollo de los instrumentos en manuales y gabinetes escolares. Se estudia el contexto en el que surge la física experimental y sus diferencias con la física escolástica hasta entonces vigente. La comparación entre ambas hace resaltar como característica más propia de la nueva física el recurso a lo empírico para exponer leyes y fenómenos, con el consiguiente abandono de la tutela de la filosofía. La irrupción de la física experimental impulsa el uso escolar de instrumentos como estrategia imprescindible de enseñanza. En el caso de España es de destacar el apoyo decisivo que recibe en el Plan Pidal (1845), que promueve la implantación de una nueva asignatura en secundaria (Elementos de física y nociones de química, $5 .^{\circ}$ curso), la publicación del primer programa de la asignatura, el anuncio de las listas de manuales e instrumentos recomendados y la creación de gabinetes en los centros escolares.

En la investigación realizada se han tenido en cuenta campos diversos como la historia de la física, la legislación educativa, los manuales de enseñanza y los instrumentos de gabinete. Así, se estudian los instrumentos que aparecen en manuales sobre una muestra seleccionada de textos significativos de universidad y de secundaria, espaciados temporalmente. Se comprueba que la presencia de instrumentos es creciente, así como de ilustraciones. El tipo de estas también evoluciona, detectándose hacia finales de siglo un máximo de las realistas frente a las más abstractas. Por otra parte, la estrecha relación de los instrumentos con el carácter experimental de un manual ha conducido a la propuesta de un índice numérico para señalar tal carácter. Aplicado a los manuales de la muestra, los resultados apuntan a que el carácter experimental es algo mayor en los manuales de secundaria que en los universitarios, aunque ambos siguen la misma pauta de evolución, con un máximo hacia el año 1880, que desciende a continuación lentamente.

El estudio considera también de modo preferente los instrumentos reales que formaron parte de los gabinetes del siglo XIX y, a este respecto, se propone un sistema de clasificación con base didáctica, en lugar del tradicional basado en la pertenencia a un área temática. Dicho sistema está constituido por diez categorías que atienden a la finalidad didáctica del instrumento (medida, estudio y demostración, modelo tecnológico, etc.). Aplicado a colecciones de varios centros escolares se constata que sus distribuciones siguen un patrón idéntico, donde destacan con más del 50\% los instrumentos de estudio y demostración. Esta distribución es semejante a la que resulta para los instrumentos que aparecen en los manuales. Por el contrario, las colecciones estudiadas que proceden de centros de investigación muestran una distribución diferente en la que predominan los instrumentos auxiliares, de medida e investigación. Se ha efectuado, además, una comparación entre las colecciones escolares antiguas y los equipos modernos (por ejemplo, ENOSA). Estos últimos destacan por un rasgo esencial, la modularidad, lo que se traduce en una distribución de perfil diferente, donde aproximadamente el $50 \%$ son instrumentos multiuso.

En cuanto a la enseñanza de la física en el periodo estudiado, parece que lo experimental solo inició un modesto despegue, sin alcanzar grandes cotas, y fue más un ideal que una realidad. A los graves problemas presupuestarios se unió la inexistencia de fabricantes nacionales de instrumentos, lo que hizo que los suministros tuvieran que venir del extranjero, especialmente de Francia. Revisando las listas oficiales y las colecciones de gabinete, destaca la unicidad de los instrumentos, lo que sugiere que la enseñanza experimental estaría reducida a experiencias de cátedra 
por parte del profesor. Por otra parte, los programas de examen conservados señalan que las cuestiones más experimentales propuestas consistían en describir instrumentos, en lugar de demostrar conocimientos acerca de su utilización. Todo lo cual apunta a una enseñanza eminentemente libresca.

Por último, el estudio de caso se centra en la catalogación y el análisis de la colección del antiguo Instituto de Granada, actual IES "Padre Suárez". Se ha inventariado la colección siguiendo las técnicas habituales. En la identificación de las piezas se han utilizado principalmente manuales de la época y catálogos de fabricantes. El resultado han sido 403 instrumentos conservados, lo que representa el $50 \%$ de la colección original, según documentos localizados en el archivo. La procedencia de los instrumentos es muy mayoritariamente de firmas comerciales francesas y su distribución por categorías corrobora el patrón característico que se ha puesto de manifiesto en los demás centros escolares.

\section{CONCEPTOS RELACIONADOS CON ESTRELLA. LINGÜÍSTICA DE CORPUS DE ASTRONOMÍA}

\author{
Autora: Cristina Silvia Hansen Ruiz \\ Tipo:Tesis doctoral \\ Director de la tesis: Dr. Jesús Pérez Ceballos \\ Departamento: Didácticas Específicas \\ Universidad: Universidad de La Laguna \\ Programa: Doctorado en Educación \\ Fecha de presentación: 5 de diciembre de 201I
}

Esta tesis se enmarca en la línea de investigación del GICEC (Grupo de Investigación de Conceptos en la Enseñanza de las Ciencias) y consta de tres partes diferenciadas: detección de los conceptos de mayor importancia de acuerdo con la frecuencia y las relaciones con las que aparecen, a través de técnicas procedentes de la lingüística de corpus; selección de conceptos y análisis de estos y de sus redes semánticas en los distintos ámbitos en los que se usan, y propuesta de enseñanza de los conceptos mediante la evaluación de recursos preexistentes o creación de estos sobre la base de los resultados del análisis de conceptos.

La investigación realizada en esta tesis se centra en la mejora y justificación de la metodología utilizada en la primera parte de la línea de investigación. La finalidad de esta metodología es la determinación de vocabularios frecuentes, vocabularios específicos, colocaciones y relaciones entre unidades léxicas. Ello requiere el uso conjunto del PAFE (Programa de Análisis de Frecuencias y Estudios de Entorno) y de técnicas de detección de vocabularios específicos, vocabularios frecuentes y colocaciones, provenientes o basadas en la lingüística de corpus. La evaluación de la metodología se ha hecho aplicándola a un caso particular de más de
1000 palabras: un texto de Astronomía realizado por el Centro de Información de la Agencia Espacial Europea del Hubble (ESA/ESO). El texto trata sobre estrellas, y se enmarca en una serie de ejercicios desarrollados para la educación secundaria. El estudio de caso se analiza con tres variaciones en la metodología: un primer análisis "ortodoxo", donde se considera todo el vocabulario del texto, es decir, se incluyen las palabras funcionales carentes de significado para el análisis de los conceptos; un segundo análisis sin palabras funcionales, y un tercer análisis donde se unifican grafías con el mismo significado, como son sinónimos o lenguaje matemático.

Los datos de frecuencia estadística de las unidades léxicas obtenidos con el PAFE son usados para proponer una forma matemática de obtener vocabularios frecuentes basados en el análisis de la distribución de frecuencias absolutas. Los vocabularios específicos se hallan comparando las frecuencias relativas del texto particular con las de un corpus general siguiendo técnicas desarrolladas en la lingüística de corpus. Además, se obtienen colocaciones comparando frecuencias y relaciones entre unidades léxicas. Las frecuencias y relaciones se usan para construir las redes semánticas enriquecidas por la información detectada previamente. 
Se observa que a la hora de usar las técnicas procedentes de la lingüística de corpus no se puede obviar la información que se encuentra en forma de lenguaje matemático, entendido este como símbolos matemáticos y conceptos que se matematizan para incluirlos en fórmulas. Algunos parámetros propios de la lingüística de corpus muestran cómo el texto elegido, que ha sido producido en una institución científica para su uso en el ámbito escolar, presenta características del lenguaje de los textos científicos y de divulgación.

Los resultados de la investigación muestran el dominio de sustantivos y adjetivos no solo en la formación de colocaciones, sino también en vocabularios frecuentes y específicos y como portadores de la mayor cantidad de información en las redes semánticas.

La investigación concluye la necesidad de: eliminar todas las palabras funcionales; unificar grafías de sinónimos solo para palabras situadas en las franjas alta y media; no eliminar el lenguaje matemático debido a su importancia tanto en frecuencias como en las redes semánticas; requerir a un experto para corregir los datos obtenidos al hallar colocaciones y vocabulario específico debido a las limitaciones de las técnicas basadas en comparaciones de las frecuencias estadísticas y para interpretar las redes semánticas; determinar matemáticamente el vocabulario más frecuente mediante el análisis de las distribuciones de frecuencias absolutas de las unidades léxicas; determinar el sistema óptimo mediante el estudio de la conservación de relaciones por cada lema además de la conservación de relaciones globales del sistema, e incorporar en las redes semánticas la información de vocabulario específico y conservación de relaciones. Además, se acotan los errores posibles en la metodología, se precisan las limitaciones que afectan a los resultados, se cuantifican los posibles errores de cada una de las fases del proceso y se determina la dependencia de la metodología respecto a la intervención de un investigador que además sea experto en el tema abordado.

Se obtiene así una metodología lista para ser aplicada sobre corpus provenientes de los géneros educativo, divulgativo y científico. Los resultados de interés para la didáctica de la ciencia obtenidos con esta metodología son: vocabularios frecuentes, vocabularios específicos y redes semánticas.

La tesis se puede consultar en la base de datos de Dialnet: <http://dialnet.unirioja.es/servlet/ tesis? codigo $=24261>$ 